\section{$\underset{\substack{\text { hommes } \\ \text { \& migrations }}}{ }$}

\section{Hommes \& migrations}

Revue française de référence sur les dynamiques

migratoires

$1314 \mid 2016$

Migrations chinoises et générations

\title{
Les associations chinoises
}

La transition générationnelle d'immigrés chinois aujourd'hui à Paris

\section{Chen Ken}

Traducteur : Stéphane Lévêque

\section{(2) OpenEdition \\ Journals}

\section{Édition électronique}

URL : http://journals.openedition.org/hommesmigrations/3629

DOI : 10.4000/hommesmigrations.3629

ISSN : 2262-3353

\section{Éditeur}

Musée national de l'histoire de l'immigration

\section{Édition imprimée}

Date de publication : 1 avril 2016

Pagination : $35-42$

ISBN : 978-2-919040-35-3

ISSN : $1142-852 X$

\section{Référence électronique}

Chen Ken, «Les associations chinoises », Hommes \& migrations [En ligne], 1314 | 2016, mis en ligne le 01 avril 2019, consulté le 20 avril 2019. URL : http://journals.openedition.org/

hommesmigrations/3629; DOI : 10.4000/hommesmigrations.3629 


\title{
LES ASSOCIATIONS CHINOISES \\ LA TRANSITION GÉNÉRATIONNELLE D'IMMIGRÉS CHINOIS AUJOURD'HUI À PARIS
}

par KEN CHEN, doctorant en sociologie, URMIS, université Paris-Diderot Paris-VII.

\author{
Créée en 2009, I'Association des jeunes Chinois de France \\ (AJCF) compte parmi les nouvelles structures qui redessinent \\ le paysage associatif chinois à Paris. Elle est l'émanation \\ du désir des descendants de migrants de mettre en avant \\ des objectifs différents de ceux de la première génération. \\ Par leur engagement socioculturel fondé sur un projet \\ de vivre ensemble, les associations de ce type entendent à \\ la fois sortir de l'entre-soi qui caractérisait les initiatives \\ de leurs aînés et poursuivre la lutte contre les préjugés qui \\ visent les Chinois en France, en s'engageant dans la politique.
}

Les associations chinoises sont de plus en plus visibles en France. Cette visibilité peut s'expliquer, entre autres, par l'engagement croissant de la nouvelle génération de Français d'origine chinoise, arrivés jeunes ou nés en France. Après avoir dressé un tableau du monde associatif chinois à Paris, nous souhaitons souligner comment la deuxième génération transforme et modernise les formes associatives, voire crée de nouvelles associations très visibles dans la sphère publique. Le cas de l'Association des jeunes Chinois de France (AJCF) ${ }^{1}$ permet de démontrer comment l'engagement associatif des jeunes est essentiel pour leur projet d'avenir, ainsi que pour leur accès à certaines sphères sociales et politiques de la société française. Les associations sont une forme d'organisation ayant pour objectif de favoriser l'agir ensemble. Les associations chinoises, comme celles créées par des immigrés d'autres origines, telles qu'elles ont été décrites dans l'ouvrage de Milena Doytcheva ${ }^{2}$, sont des groupements affinitaires conçus pour défendre des intérêts et des buts communs en rapport avec une condition sociale marquée par l'expérience de la migration et de l'altérité. En outre, l'investissement associatif est un mode d'entrée dans la sphère publique, un moyen d'exprimer collectivement des opinions, une forme de socialisation et d'apprentissage du fonctionnement de la vie collective. 


\section{Typologie des associations chinoises à Paris}

La consultation des données des maisons d'association ainsi que du Carrefour d'associations parisiennes (CAP) a permis de faire une estimation du nombre des associations chinoises à Paris (sans compter celles qui ne sont pas déclarées). Il y aurait, aujourd'hui, plus de 700 associations chinoises, ou franco-chinoises. Les associations chinoises, dont le bureau, le conseil d'administration et l'essentiel des membres sont composés de Chinois ou de personnes d'origine chinoise, sont de plusieurs types. Premièrement, les asso-

En revanche, les jeunes leaders français d'origine

chinoise, qui ont créé

leurs propres associations,

remettent en cause

l'accaparement du pouvoir par les "vieux". ciations géographiques, créées sur la base d'une région d'origine commune. Elles sont appelées "Tongxianghui" en chinois. Elles visent à resserrer les liens d'une communauté issue de la même origine en assurant la solidarité et l'entraide. En France, les plus connues sont l'Association des Chinois de Qingtian et l'Association des ressortissants de Chaozhou. Deuxièment, les associations professionnelles sont des associations créées en fonction des métiers des migrants chinois, telles l'Association des commerçants et industriels franco-chinois, l'Association des écrivains chinois, l'Association des commerçants chinois du prêt-àporter en France, etc. Troisièmement, les associations d'anciens étudiants (alumni) visent à créer une solidarité entre des membres issus du même établissement scolaire chinois ${ }^{3}$. Par exemple : l'Association des anciens élèves de SJTU en France, l'Association des anciens élèves de l'université Tsinghua en France. Quatrièmement, les associations religieuses sont surtout des associations bouddhistes et chrétiennes, en particulier protestantes ${ }^{4}$, quoique quelques groupes catholiques chinois s'or- ganisent aussi autour de l'église Saint-Hippolyte du XIII arrondissement ou de l'église Sainte-Élisabethde-Hongrie du III arrondissement ${ }^{5}$. Enfin, les associations créées pour répondre aux besoins socioculturels et politiques des immigrés chinois sont des associations plus récentes, qui répondent à lévolution de la population telles que l'AJCF (dont nous parlons ci-dessous), Chinois de France-Français de Chine (CFFC), qui propose des aides concrètes dans le quartier de Belleville, le Conseil représentatif des associations asiatiques de France (CRAAF), qui vise à promouvoir la participation politique. Cette dernière catégorie d'associations se concentre sur des activités favorisant l'intégration telles que les cours de langue, les événements sportifs, l'orientation et la formation professionnelles. Ces associations s'ouvrent plus souvent sur la société d'accueil et représentent une porte pour la reconnaissance des migrants chinois. Les membres y sont plus divers : Chinois, descendants de Chinois et autres Français ou immigrés s'y rencontrent.

\section{Associations chinoises et générations}

Les entretiens réalisés auprès de représentants d'associations chinoises, telles que l'AJCF et CFFC, mettent en évidence les caractéristiques différentes des associations des immigrés chinois et de celles de leurs descendants. Les associations chinoises créées par la première génération tendent à circonscrire leurs actions dans un champ limité. Les contacts avec la population locale, française ou issue d'autres origines, se réduisent aux interactions commerciales et administratives. Ces associations chinoises se présentent comme les défenseurs légitimes des droits de leurs compatriotes et comme des médiateurs pour la résolution des conflits internes. L'objectif des associations créées par de jeunes Français d'origine chinoise est plutôt d'aider à la réalisation de projets, professionnels en particulier. Sans 
Entraînement de Wushu, art martial traditionnel chinois, suivi par des jeunes de l'Association des commerçants bellevillois. Place Marcel Achard. Paris XXe, mai 2016. (C CAmille Millerand

renier leur identité chinoise, ils souhaitent ouvrir leurs associations aux autres et s'inspirent des pratiques et d'idées auprès d'autres associations ${ }^{6}$. Par ailleurs, les relations au sein des associations ont également évolué avec le changement de génération. Les leaders des associations de la première génération sont décrits comme dirigistes. Leur comportement rappelle les rapports d'autorité qui peuvent régner entre les générations. Ces leaders sont très souvent de riches marchands, des notables qui mettent leur aura et leurs ressources au service de la commu- nauté et deviennent des référents. En revanche, les jeunes leaders français d'origine chinoise, qui ont crée leurs propres associations, remettent en cause l'accaparement du pouvoir par les "vieux". Ces revendications semblent d'autant plus légitimes que les associations requièrent souvent leurs compétences (maîtrise orale et écrite de la langue française, connaissance des rouages de la société d'accueil, partenariat avec divers organismes, etc.). Néanmoins, malgré les tensions intergénérationnelles, la coopération existe entre les deux générations d'associations ${ }^{8}$.

6. Carine Pina-Guerassimoff, La Chine et sa nouvelle diaspora. La mobilité au service de la puissance, Paris, Ellipses, 2012.

7. François Leung, Xingqui Ye, Brève histoire de la communauté chinoise en France, Paris, éd. Pacifica, 2014.

8. Sur la complémentarité des générations au sein des associations, voir François Leung, XingquiYe, op. cit., mais aussi le cas

des associations comoriennes en France décrit par Vivier Géraldine, "Les associations comoriennes en France : processus migratoires, identités et transformations sociales", in Hommes\&Migrations, $n^{\circ} 1226$, 2000, pp. 58-74. 


\section{Associations chinoises et reconnaissance publique}

Les activités des associations chinoises ont beaucoup de valeur aux yeux des élus qui soutiennent de plus en plus souvent leurs demandes et témoignent de la qualité du travail associatif. La députée et vice-présidente déléguée du parti Les Républicains, Nathalie Kosciusko-Morizet, ainsi que le président de l'Assemblée nationale, Claude Bartolone, ont participé à une soirée en l'honneur de l'implication des jeunes

À cela, il convient de rajouter

la barrière de la langue qui provoque, chez les immigrés

chinois, le sentiment que les autorités françaises ne s'intéressent pas à leur vie quotidienne ni à leur conditions sociale et politique.
Français d'origine chinoise dans la société française en novembre dernier'.

Beaucoup de présidents d'associations chinoises insistent sur l'importance d'“améliorer la participation politique des immigrés chinois en France ${ }^{10 "}$, comme le souligne monsieur

$S$, président du CRAAF et propriétaire de restaurants au centre de Paris ${ }^{11}$. Par exemple aujourd'hui, des responsables d'associations chinoises siègent au sein des conseils d'administration d'arrondissement où résident une grande part d'immigrés chinois ou de Français d'origine chinoise, comme le III et le XXe arrondissement, et portent des revendications favorisant les Chinois. Les associations se mobilisent également par des actions symboliques avec les autorités locales. La visite des élus et d'autres personnalités locales, lors des activités des associations, témoigne des relations de reconnaissance. Ces échanges, régis par les logiques politiques, sont dans l'intérêt des résidents chinois, mais aussi des représentants politiques, dans la mesure où leur soutien rend visible, aux yeux des électeurs, leur bonne gestion de la cohésion sociale. Pourtant, les entretiens et notes de terrain soulignent que les initiatives locales visant les immigrés chinois sont encore peu nombreuses, car les autorités ont du mal à bien cerner cette population qui s'exprime particulièrement peu sur la scène publique. Ce phénomène n'est pas spécifique à la France et a été observé ailleurs, comme pour les immigrés chinois aux États-Unis ${ }^{12}$. À cela, il convient d'ajouter la barrière de la langue qui provoque, chez les immigrés chinois, le sentiment que les autorités françaises ne s'intéressent pas à leur vie quotidienne ni à leur condition sociale et politique. Certains d'entre eux, notamment les plus âgés, issus de la première génération d'immigrés, perdent l'envie de s'exprimer : "Les autorités ne nous écoutent pas souvent, alors j'arrête, et je gagne l'argent pour ma famille ${ }^{13}$, comme le déclare un immigré chinois de 55 ans, vivant depuis plus de trente ans en France.

Ainsi, les associations jouent le rôle de ponts entre la population chinoise et les services publics. Elles sont un support important des autorités françaises, puisque les activités qu'elles proposent en faveur de la formation à l'emploi, à la langue, au soutien scolaire, à la santé, ainsi qu’aux démarches administratives, complètent la gestion publique de ces enjeux. Elles reçoivent pour cela de plus en plus de financements publics. Les associations chinoises ne sont d'ailleurs pas pionnières en la matière. Les associations d'immigrés africaines ${ }^{14}$, maghrébines ${ }^{15}$ et portugaises ${ }^{16}$ ont joué le même rôle avant les associations chinoises, et ont inspiré ces dernières.

\section{L'AJCF : une association de la deuxième génération}

Les Chinois de France entrent aujourd'hui dans une phase de prise de conscience de leur propre réalité sociale au sein de la culture française et en miroir

9. Voir Sihem Souid, “Les Français d’origine chinoise s'organisent pour peser en politique”, in Le Point, 24 septembre 2015. 10. Entretien avec M.S, novembre 2014, restaurant à Châtelet. 11. http://craaf.info/_12. Min Zhou, Contemporary Chinese America: Immigration, Ethnicity, and Community Transformation, Philadel phie, Temple University Press, 2009. 13. Entretien avec M. Gao, décembre 2014, rue de Belleville. 14. Pierre Kamdem, Le Mouvement associatif de la diaspora camerounaise. Enjeux et perspectives, Paris, L'Harmattan, 2008. 15. Dominique Baillet, “Jeunes d'origine maghrébine dans l'espace associatif et politique”, in Migrations Société, vol. 11, n66, 1999, pp. 7-22. 16. Jorge de La Barre, Jeunes d’origine portugaise en association. On est européen sans le savoir, Paris, L’Harmattan, 1997. 
avec les autres populations issues de l'immigration. Les associations chinoises se tournent alors vers l'espoir d'un développement des échanges interculturels dans le respect des différences ; les participants sont plus désireux d'ouverture et les associations promeuvent le contact avec les autres. " $\mathrm{Ce}$ n'est pas dans le conflit ou dans l'incompréhension que les bonnes relations s'établissent, mais dans l'échange durable", selon le directeur de l'AJCF ${ }^{17}$. L'association a été créée en 2009 par des jeunes Français d’origine chinoise, le plus souvent nés en France mais qui gardent une forte relation avec la Chine et les Chinois. Sacha Lin, ancien président et membre fondateur de l'AJCF raconte comment l'association a été créée: "Nous voulions créer un outil social pour les jeunes Français-Chinois, construire un espace indépendant de discussion et d'expression autour d'un projet associatif et un lieu de partage, où les jeunes Français d'origine chinoise viennent trouver les ressources nécessaires à leur épanouissement. On voulait encourager les jeunes citoyens à se rendre utiles au sein de la société $e^{18}$." L'indépendance qu'il mentionne renvoie au désir de prendre de l'autonomie par rapport aux grandes associations chinoises et aux leaders de l'ancienne génération, cela dans le but de favoriser d'autres types de fonctionnement et d'action. Dans cette citation, trois points importants méritent d'être développés : les relations intergénérationnelles, l'engagement socioculturel et enfin l'engagement politique.

\section{Prise d'autonomie et coopération avec les leaders de la première génération}

Contrairement à la majorité des associations de la première génération, que nous avons décrites cidessus, les membres et sympathisants de l'AJCF ne se retrouvent pas sur la base d'un lieu de naissance ou sur des origines ou une profession communes, mais sur la base de valeurs, d'une histoire, d'aspirations, de vécus, de déceptions ou de succès parta- gés. Le point commun entre eux est le succès, car les membres de l'association ont plus ou moins tous connu une réussite professionnelle ou économique. Leurs métiers sont diversifiés : avocat, comptable, commerçant qualifié et innovant, ingénieur, etc. Par conséquent, leurs objectifs sociaux et politiques sont plus ambitieux afin d'améliorer le statut de la population chinoise en France. Quoique désireux de se distinguer des associations plus historiques, dirigées par des leaders des générations précédentes, la coopération avec les grandes associations reste
Plus que les associations de la première génération, les associations à faire des diverses manifestations des lieux de promotion des sensibilités de l'immigrant, mais aussi des espaces de communication. de descendants cherchent importante. En conséquence,

l'AJCF invite régulièrement des représentants connus des associations plus anciennes à participer aux événements visibles (telle la fête de la Lune ou le défilé du Nouvel An chinois). En particulier, l'AJCF valorise la présence de leaders de l'ancienne génération qui ont travaillé avec les autorités locales afin de promouvoir l'image et la reconnaissance des immigrés chinois en France. Certaines grandes associations de la première génération d'immigrés chinois ont également reconnu les compétences de l'AJCF. Des leaders ont proposé leur coopération ou un soutien financier à ces jeunes diplômés, qui connaissent bien la société française et les pratiques associatives. Ils les soutiennent dans leurs objectifs de visibilité médiatique et d'ascension sociale, ce qui était difficile pour les leaders d'association de la première génération limités par leur niveau linguistique et leur connaissance de la culture française.

\section{L'engagement socioculturel}

Les activités culturelles sont souvent la raison d'être des associations. Les activités abordent des thématiques en lien avec le pays d'origine : l'objectif de l'AJCF est de "favoriser une meilleure compréhension de l'immigration chinoise en France, de faire décou- 
les associations de descendants cherchent à faire des diverses manifestations des lieux de promotion des sensibilités de l'immigrant, mais aussi des espaces de communication ${ }^{21}$. Les jeunes d'origine chinoise privilégient la communication avec autrui pour éviter la fermeture sur soi et comme moyen de reconnaissance. C'est le cas de l'AJCF qui a progressivement pris de l'importance grâce aux interventions dans les médias, aux activités ouvertes sur les quartiers, aux conférences, ainsi qu'au développement de partenariats avec des institutions publiques et d'autres associations. Les membres et participants de l'association sont aujourd'hui issus de différentes origines chinoises et non chinoises.

L'AJCF contribue en premier lieu au développement d'un meilleur vivre ensemble par la déconstruction de certains clichés. Le vivre ensemble suppose la connaissance et la conscience des diversités sans que ce soit un frein au bien-être des différentes communautés ${ }^{22}$ et générations ${ }^{23}$. L'association s'emploie donc à créer des échanges entre personnes d'origines et de générations différentes au cours "des projections-débats, des conférences, des dîners-débats ou encore des événements plus singuliers, comme des concerts franco-chinois ${ }^{24 "}$. L'AJCF mène également

Olivier Chen en terminale au lycée Bergson ( XIXe à Paris) anime une séance de musculation au sous-sol de l'Association des commerçants bellevillois, un entraînement fréquenté aussi par des jeunes d'autres communautés, mai 2016. o Camille Millerand

vrir la culture chinoise et de multiplier les échanges et interactions avec la Chine contemporaine $e^{19}$. Parmi les activités, les manifestations culturelles sont privilégiées car elles permettent de marquer sa présence dans les espaces publics, de valoriser sa culture ${ }^{20}$. Plus que les associations de la première génération, des actions de terrain pour répondre au mieux aux besoins des résidents : soutien scolaire, mobilisation lors des réunions de quartier concernant les questions sociales. L'association travaille aussi à la médiatisation du travail souvent méconnu de personnalités locales, des associations, des bénévoles du quartier ou des chercheurs, au bénéfice des immigrés chinois dans leur vie quotidienne. Cette médiatisation offre une reconnaissance et permet de "partager les expériences des bénévoles, pour accompagner chacun à aller plus loin ${ }^{25 "}$.

20. Antoine Dumont, "S'associer pour se souvenir. Le travail de mémoire des associations de migrants marocains en France", in Diasporas. Histoire et Sociétés, $n^{\circ}$ 6, 2005, pp. 96-108. 21. Alain Battegay, Ahmed Boubeker, "L'action associative des jeunes Maghrébins entre affirmation d'un endroit cité et pratiques urbaines", in Les Annales de la recherche urbaine, $\mathrm{n}^{\circ} 89,2001$. 22. Thierry Leterre, "L'art de vivre ensemble. Science politique et sociologie chez Comte", in Archives de philosophie, vol. 1, $n^{\circ} 70,2007$, pp. 77-93. 23. Laurent Thévenot, “Une science de la vie ensemble dans le monde”, in Revue du MAUSS, vol. 2, n² 24,2004 , pp. 115-126. 24. Citation issue du site AJCF : WWw.lajcf.fr, rubrique “Activités". 25. Entretien avec le directeur de l'AJCF, mai 2015, dîner mensuel de l'AJCF. 


\section{L'AJCF en lutte contre le racisme}

Au-delà de l'engagement socioculturel, l'AJCF porte aussi la voix des Français d'origine chinoise sur la scène publique et cela grâce à trois types d'action collective : le recours à la presse, au système judiciaire et aux mobilisations de rue. Ce type d'actions permet aux immigrés d'exprimer leurs revendications ${ }^{26}$. Par l'action collective, ils cherchent à défendre leur sécurité, l'égalité de traitement ou l'ascension de leur statut social et politique. Ils cherchent aussi à dénoncer l'indifférence et l'inaction des autorités ${ }^{27}$.

L'AJCF a participé au comité d'organisation de la manifestation contre l'insécurité à Belleville en 2011, qui était une action symbolique d'immigrés chinois en France revendiquant le besoin d'une action renforcée pour garantir leur sécurité dans le quartier de Belleville.

L'AJCF s'est, par ailleurs, portée partie civile dans un procès contre le journal Le Point pour dénoncer des discours racistes visant les Chinois. En 2012, l'association s'est engagée dans une procédure juridique en coopérant avec l'association SOS Racisme contre le magazine qui a publié le 23 août 2012 un article intitulé "L'intrigante réussite des Chinois en France" dépeignant le parcours décrit comme typique de l'immigrant chinois, de la clandestinité à la réussite ${ }^{28}$.

Cet article inventait notamment "les 5 commandements de l'entrepreneur chinois", une synthèse "scandaleuse" de préjugés racistes, selon l'AJCF. Le vendredi 24 janvier 2014, le tribunal correctionnel de Paris a jugé diffamatoires trois des cinq "commandements": "Tu ne rémunéreras pas tes employés car ce sont des membres de ta famille", "Tu ne cotiseras pas et donc tu ne toucheras pas d'aides" et "Tu ne paieras pas d'impôts ${ }^{29 "}$ ". Par conséquent, le directeur de la publication, Franz-Olivier Giesbert, a été condamné à 1500 euros d'amende pour "diffamation publique envers un groupe de personnes en raison de leur origine ou nationalité chinoise $e^{30}$. "C'est la première fois en France qu'une plainte est déposée pour une diffamation raciale visant les Français d'origine chinoise ${ }^{31 "}$ ", a expliqué Rui Wang, porte-parole de l'AJCF, qui est devenu le président actuel de l'association.

Il est à noter que la présence de l'association AJCF dans les médias se multiplie depuis quelques années; les médias français et chinois, tels que $\mathrm{RFI}^{32}$ et $\mathrm{L}^{\prime} \mathrm{Obs} s^{33}$, ont publié des articles sur l'association. Les dirigeants ont participé à des interviews à la radio où ils ont pu préciser leurs idées et leurs perspectives ${ }^{34}$, surtout après l'affaire du journal Le Point, qui marque un tournant dans le traitement par la presse des immigrés chinois et le Les associations chinoises ont contribué à former une élite intellectuelle et politique contestataire recul des préjugés et des propos d'origine chinoise. racistes. Ces préjugés sont très présents, comme le raconte Maryline Zheng, étudiante en droit, vice-présidente de l'association AJCF dans un entretien pour France Info : "Moi, ce qu'on me dit souvent, c'est que le Chinois, c'est de la mauvaise qualité, ce ne sont que des vendeurs de nems, des mafieux, des prostituées, de l'argent opaque. Tous les jours on nous renvoie ces clichés. Pour quelqu'un comme moi qui suis née en France, qui ai étudié à l'école de la République, c'est très violent, très choquant ${ }^{35}$." Elle invite dans cet entretien les Chinois à faire entendre leur voix, à participer à la vie publique, à s'inscrire sur les listes électorales et à dire quand ils ne sont pas d'accord.

26. Olivier Fillieule, Danielle Tartakowsy, La Manifestation, Paris, Presses de Sciences Po, 2004.

27. Lilian Mathieu, Comment lutter? Sociologie et mouvements sociaux, Paris, Textuel, 2004. 28. Le Point a retiré son article

en ligne suite à la condamnation. II est en lecture sur le lien suivant : lachineaparis.fr/uploads/Le Point 23 août.

29. Voir Le Point condamné pour diffamation pour un article sur les immigrants chinois", in Le Monde.fr, 25 janvier 2014

30. Ibid. 31. Voir "Giesbert poursuivi pour diffamation", in LeFigaro.fr, 10 décembre 2012.

32. Voir "Chinatown: a neighborhood known worldwide", in RFI.fr, 31 janvier 2013. 33. Bernard Genies, "Les Chinois de Paris.

Enquête sur une nouvelle génération", in Le Nouvel Observateur, 15 janvier 2014.

34. Mathilde Lemaire, "Qui sont les 600000 Chinois de France?", in Franceinfo.fr, 27 janvier 2014.

35. Ibid 


\section{De l'association à l'engagement politique}

Les associations chinoises ont contribué à former une élite intellectuelle et politique contestataire d'origine chinoise. Déjà bien intégrés à la société française grâce à leur diplôme élevé et leur profession souvent prestigieuse, les leaders de l'AJCF ont consolidé leur position grâce à la vie associative qui leur a permis de créer des relations avec les autorités locales de leurs arrondissements. Par exemple, Olivier Wang, qui est l'un des fondateurs de l'association AJCF, est devenu maire adjoint du XIXe arrondissement lors des élections municipales de $2014^{36}$. Depuis la création de l'association en 2009, il avait notamment soutenu les commerçants chinois à Paris. Il était le porte-parole de la manifestation des immigrés chinois contre l'insécurité à Belleville en 2011. En entrant dans la vie politique française, il est devenu un symbole pour les Chinois de France,.

\section{L'association comme ressource professionnelle}

D'après le président actuel de l'AJCF, il faut "avouer" néanmoins que les jeunes d'origine chinoise ont aussi des objectifs personnels en s'engageant dans des associations, car "personne ne travaille pour rien, les bénévoles ont quand même leurs intérêts 37" $^{37}$ Les associations chinoises, comme d'autres associations de migrants et de leurs descendants, sont un lieu propice pour faire connaissance et créer des réseaux personnels et professionnels. Certains membres tirent de leur expérience associative un capital culturel et professionnel qui peut leur permettre d'accroître leur capital économique ${ }^{38}$. D'autres parviennent à créer leurs propres entreprises dans le secteur privé en utilisant des ressources de l'association (sous la forme de formation professionnelle, par exemple) et en tissant des liens privilégiés avec des personnes qui deviendront des collaborateurs ${ }^{39}$.

L'intérêt personnel est une motivation incontournable pour que certains participants s'engagent dans les activités, avec le désir de rencontrer ceux qui possèdent un capital économique, culturel et social ${ }^{40}$. Toutefois, le rôle d'entraide de l'association reste central, même lorsqu'il s'agit de carrière, ainsi que l'illustre la création d'un service de moniteur entrepreneurial, il s'agit d'“un soutien technique particulièrement utile dans le contexte de la mondialisation, ils doivent créer plus de liens dans différents lieux afin de se promouvoir ${ }^{41}$ ".

\section{Conclusion}

Grâce à leur niveau de scolarisation et à leur expérience professionnelle, les descendants d'immigrés chinois ont fait progresser la vie associative chinoise en termes d'organisation et d'engagement. En effet, pour la première génération, les associations chinoises sont plutôt un lieu d'entre soi où se consolide la solidarité au sein du groupe. En revanche, pour les jeunes, les associations chinoises sont devenues un dispositif qui les aide à réaliser leurs projets dans la société plus large. Les nouveaux dirigeants d'associations et leurs membres se concentrent davantage sur la communication vers l'extérieur, via les activités interculturelles et l'engagement politique, ce qui leur donne plus de visibilité sur la scène publique en France. 